\title{
A compact Germanium spectrometer for nuclear astrophysics
}

\section{Zachary Hughes, ${ }^{a, *}$ Manel Errando ${ }^{a}$ and William Ho ${ }^{a}$}

${ }^{a}$ Department of Physics, Washington University in St. Louis, St. Louis, MO 63130, USA

E-mail: zdhughes@wustl.edu

Emission from electron-positron annihilation at $511 \mathrm{keV}$ was the first extrasolar gamma-ray line ever detected. Despite more than 30 years of theoretical and observational progress, the origin of the positron population has yet to be identified, with potential candidates ranging from microquasars and X-ray binaries to annihilation or decay of dark matter particles. At energies between $200 \mathrm{keV}$ and several $\mathrm{MeV}$, where positron annihilation and most other gamma-ray lines of interest are located, the largest source of instrumental background are secondary protons, neutrons, and photons produced by the spacecraft when it is irradiated by cosmic rays in a space environment. This background is the main factor limiting the sensitivity of current gamma-ray spectrometers, and is proportional to the amount of mass around the detectors. We present progress towards a compact, modular, high-purity Germanium spectrometer that can be integrated into future astrophysics payloads and be the basis of small-satellite missions. A CubeSAT or SmallSAT-class mission based on compact spectrometer modules could have up to $30 \%$ of the total spacecraft mass in active germanium crystal, compared to $0.6 \%$ in current missions like INTEGRAL, leading to more than an order-of-magnitude improvement in signal-to-noise ratio due the reduction in overall spacecraft mass.

$37^{\text {th }}$ International Cosmic Ray Conference (ICRC 2021)

July 12 th - 23rd, 2021

Online - Berlin, Germany

\footnotetext{
${ }^{*}$ Presenter
} 


\section{Overview}

Studies of the $511 \mathrm{keV}$ positron annihilation gamma-ray line have yet to identify the origin of the population of galactic positrons. The SPI instrument on board INTEGRAL has provided the most complete mapping of the $511 \mathrm{keV}$ sky to date. The map reveals an emission morphology unlike that found at other wavelength. A recent SPI-derived model (Fig. 1) shows a bright, extended bulge along with a low surface brightness disk component extending $21^{\circ}$ in latitude and a high bulge-to-disk ratio [1]. Neither the shape of the bulge nor the surface brightness are currently well determined. Multiple origins for this signal have been proposed including $\beta^{+}$emission from the decay of unstable nuclei produced by massive stars or stellar explosions, high-energy particle interactions of cosmic rays with the interstellar medium, compact objects such as millisecond pulsars or microquasars, and the decay of ${ }^{56}$ Co during Type-I supernovae [2]. More tantalizingly, the broad $21^{\circ}$ disk extent has been suggested as a possible signal originating from the annihilation or decay of dark matter particles in the galactic halo. Despite these multiple competing models, no conclusive candidate explanation has emerged.

The limited $16^{\circ}$ field of view of INTEGRAL-SPI is an inherent limitation to developing a more complete understanding of the nature of the $511 \mathrm{keV}$ sky. A large fraction of the exposure of INTEGRAL has been dominated by pointings towards the galactic center and along the inner galactic disk, while exposure at high galactic latitudes remains limited (Fig. 2). An all-sky instrument capable of mapping high galactic latitudes would provide a major step forward in advancing the under-

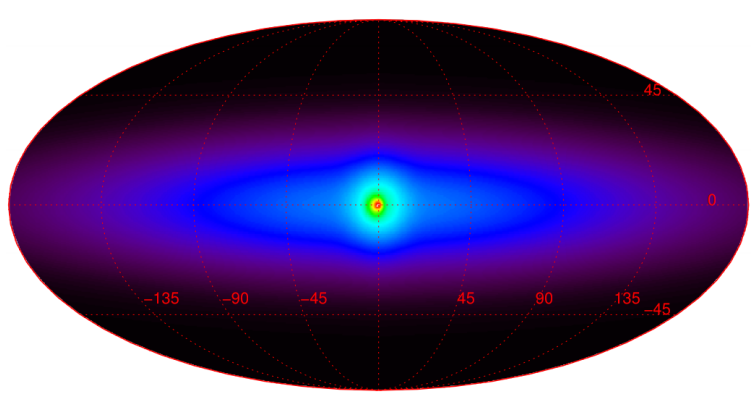

Figure 1: The best-fit spatial components model map of SPI $511 \mathrm{keV}$ data [1]. standing of current models and potentially lead to new positron sources. The COSI balloon flight in 2016 provided a more uniform coverage of the southern sky but had a sensitivity limited by a 46-day flight duration [3, 4].

An inherent additional challenge in the $511 \mathrm{keV}$ domain is the high instrumental background caused by cosmic ray induced radioactivity in the germanium detector and the surrounding spacecraft material. Figure 3 shows that this instrumental background can be dominant between $200 \mathrm{keV}$ and $2 \mathrm{MeV}$. Such background is proportional to the surrounding instrument mass [5]. A path to increasing total sensitivity, therefore, is a mission design that maximizes the mass of the germanium spec-

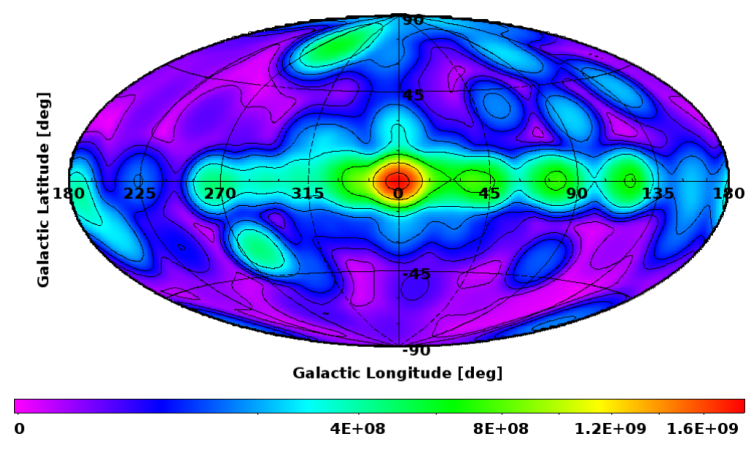

Figure 2: INTEGRAL all-sky exposure map, from [1]. trometer for gamma-ray collection efficiency while minimizing the passive support structure of the overall instrument to reduce instrumental background. By maximizing the detector's active mass fraction, over an order of magnitude in- 
crease in sensitivity is possible. A CubeSAT or SmallSAT mission provides a natural basis for such a mission due to industry-induced pressure to design integrated, light-weight, low-power platforms with integrated buses, attitude control, solar panels, and batteries. Using such a platform, a prototype spacecraft can be designed where up to $30 \%$ of the total spacecraft mass is active detector. For reference, the germanium detectors in INTEGRAL represent only $0.6 \%$ of the total spacecraft mass.

\section{Prospective detector design}

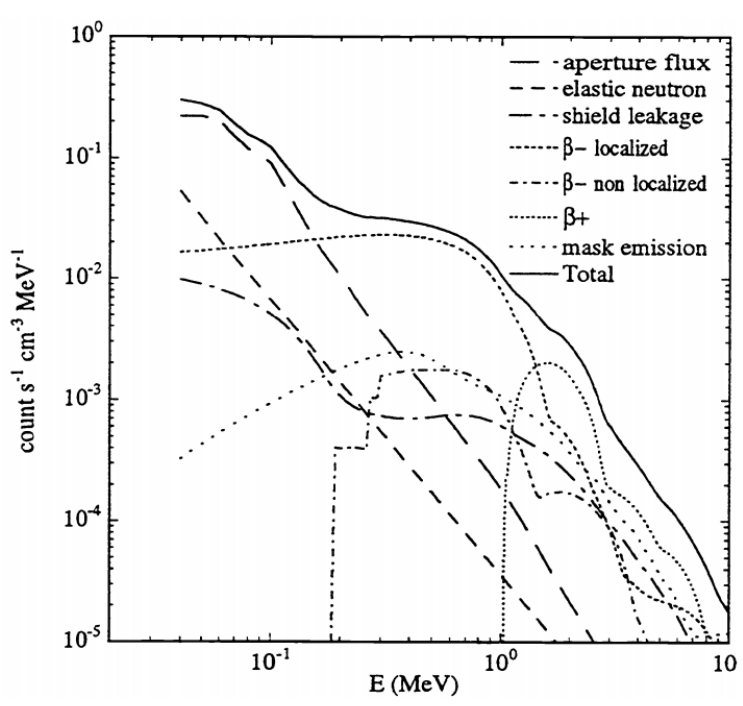

Figure 3: Predicted background to the INTEGRAL SPI instrument and its constituent components [5].
A potential implementation of a CubeSATclass mission that optimizes for an active detector mass fraction consist of six $56 \times 53 \mathrm{~mm}$ cylindrical germanium modules in a $12 \mathrm{U}$ formfactor. A necessary requirement to achieve the desired $2 \mathrm{keV}$-at-511 keV energy resolution is operating the germanium detectors at temperatures below $100 \mathrm{~K}$. This can be accomplished with existing space-qualified cryocoolers suck as the Space Micro Pulse Tube Cooler by Northrop Grumman [6] while still fitting within the size and power envelops of a CubeSAT-class mission. Further mass reduction can be accomplished if by implementing an alternative imaging technique than coded masks. On viable alternative is the occultation technique, in which direction reconstruction of the gamma-ray signal is accomplished by measuring changes in the event rate as it orbits its occulting body, either the Earth or the Moon [7]. Such techniques been successfully used on previous experiments, including BATSE [8] and the Fermi Gamma-ray Burst Monitor [9]. To reduce the background from direct energy deposition from cosmic-ray events, an anticoincidence shield consisting of a plastic scintillator along with a CsI (or similar) crystal along the back of the detector to reject rear- entering gamma rays. Figure 4 shows a schematic of both a single module and the occultation technique used for source direction determination. A CubeSAT based on this design would could achieve effective areas of $\sim 60 \mathrm{~cm}^{2}$ at $511 \mathrm{keV}$ (Figure 6), but have comparable sensitivity to INTEGRAL $\left(\sim 90 \mathrm{~cm}^{2}\right)$ due to its order-ofmagnitude lower background; while maintaining a comparable angular resolution and acting as an all-sky instrument. 

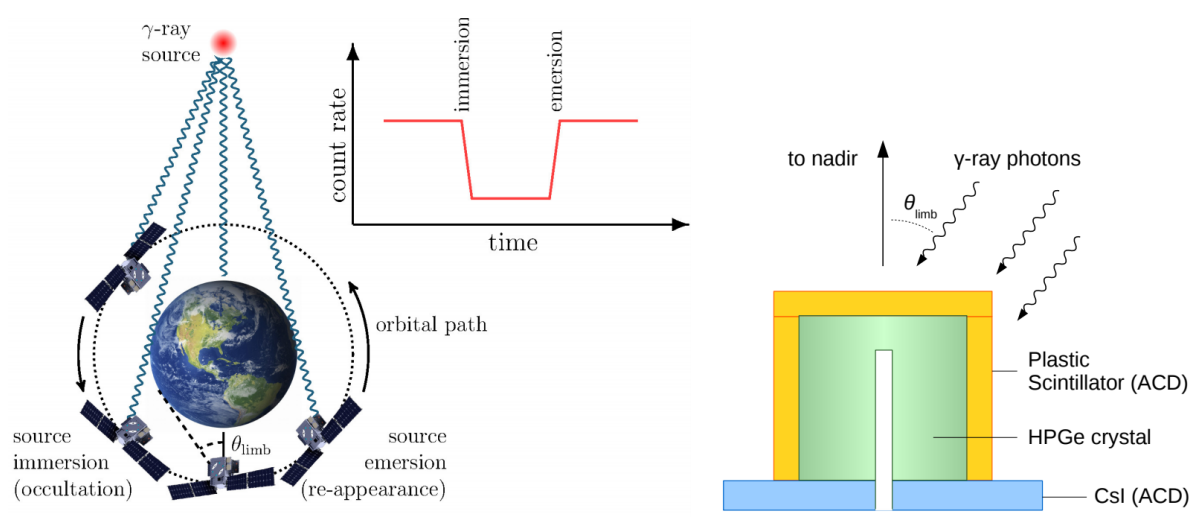

Figure 4: Left: Diagram showing the conceptual process of an occultation-imaging spacecraft. Right: Cross-section of an individual germanium module that would form the basis of the cubeSAT mission.

\section{Simulation}

Preliminary simulation of an array of germanium modules and their performances was performed. A hexagonal array of seven $56 \times$ $53 \mathrm{~mm}$ germanium modules was simulated and the detector's effective area at $511 \mathrm{keV}$ was calculated as a function of incident zenith angle. Figure 5 shows the detector layout of this simulation.

The detector simulation was run using the MEGAlib package, a suite of software tools designed to simulate and analyze gamma-ray detectors in the Compton regime [10]. The initial detector geometry was constructed in Geomega and imported into the Geant4-based Cosima program for calculating the detector response to a monoenergetic $511 \mathrm{keV}$ disk emitter and a $2 \mathrm{keV}$ HPGe energy resolution. The simulation

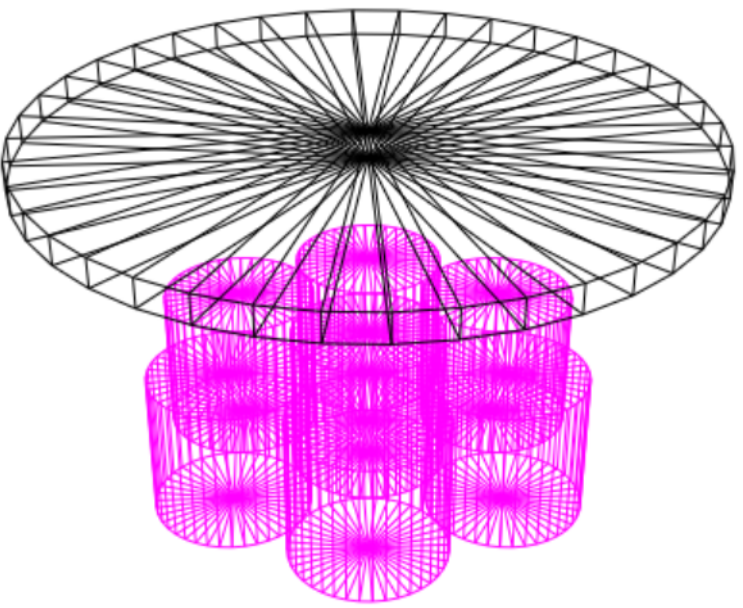

Figure 5: Geomega rendering of the basic HPGe simulation geometry used in the effective area calculations. The black disk indicates the simulated source of gamma rays for calculation of the effective area. output is then analyzed using Revan - real event analyzer - for the reconstruction of the event spectrum and from which the simulated effective area is calculated. Figure 6 and 7 show the effective area as a function of zenith angle and the resulting detector energy spectrum, respectively. The specific detector geometry can be optimized based on the spacecraft's limb angle with the occulting body, which will depend on the orbital altitude of the mission. 


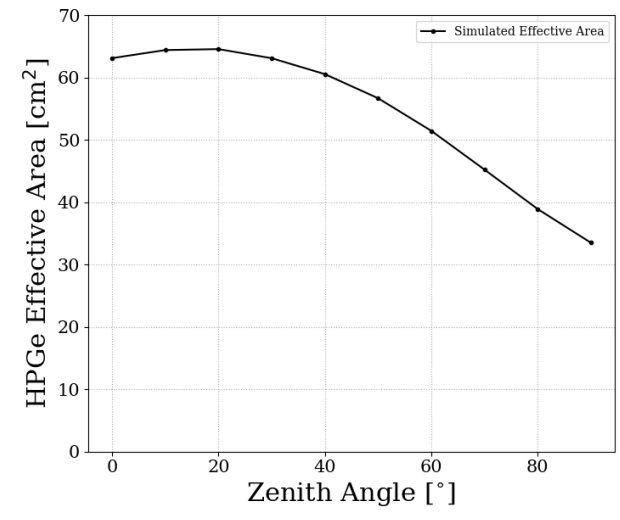

Figure 6: Effective area calculation of the 7-module HPGe at varying zenith angle.

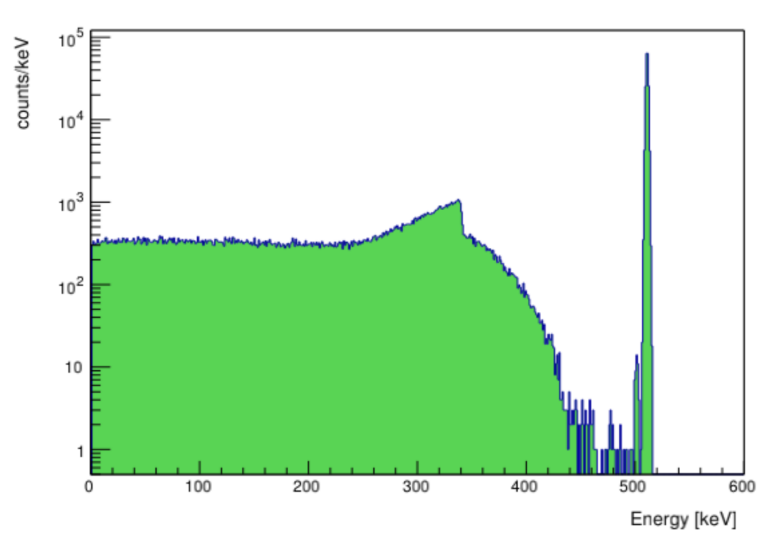

Figure 7: Energy spectrum of the HPGe subject to a $511 \mathrm{keV}$ beam.

\section{Summary}

The development of a compact, all-sky, high energy resolution instrument utilizing HPGe for gamma-ray spectroscopy is both achievable using currently available technologies and has the potential to provide an order-of-magnitude increase in sensitivity over the comparable INTEGRAL spacecraft. The increased sensitivity and all-sky coverage of such an instrument would materially advance our understanding of the $\mathrm{kMeV}$ sky, and in doing so provide an important multimessanger probe for cosmic rays, dark matter, stellar nucleosynthesis, and the physics of compact objects. Driven by industry pressure, the quickly maturing CubeSAT and SmallSAT platforms provide the natural basis for such a mission and can provide decreased costs, complexity, and time investment compared to traditional development cycles.

\section{References}

[1] T. Siegert, R. Diehl, G. Khachatryan, M.G.H. Krause, F. Guglielmetti, J. Greiner et al., Gamma-ray spectroscopy of positron annihilation in the Milky Way, Astronomy and Astrophysics 586 (2016) A84 [1512.00325].

[2] C.A. Kierans et al., Positron Annihilation in the Galaxy, 1903.05569.

[3] C.A. Kierans, S.E. Boggs, A. Zoglauer, A.W. Lowell, C. Sleator, J. Beechert et al., Detection of the 511 keV Galactic Positron Annihilation Line with COSI, Astrophysical Journal 895 (2020) 44 [1912.00110].

[4] T. Siegert, S.E. Boggs, J.A. Tomsick, A.C. Zoglauer, C.A. Kierans, C.C. Sleator et al., Imaging the 511 keV Positron Annihilation Sky with COSI, Astrophysical Journal 897 (2020) 45 [2005 . 10950].

[5] P. Jean, P. von Ballmoos, G. Vedrenne and J.E. Naya, Performance of advanced Ge-spectrometer for nuclear astrophysics, in Gamma-Ray and Cosmic-Ray Detectors, Techniques, and Missions,

B.D. Ramsey and T.A. Parnell, eds., vol. 2806 of Society of Photo-Optical Instrumentation Engineers (SPIE) Conference Series, pp. 457-471, Oct., 1996, DOI. 
[6] T. Nguyen, M. Petach, M. Michealian, J. Raab and E. Tward, Space Micro Pulse Tube Cooler, Tech. rep. (2010).

[7] R.S. Miller and D.J. Lawrence, FIRST LIGHT: MeV ASTROPHYSICS FROM THE MOON, The Astrophysical Journal 823 (2016) L31.

[8] C.A. Wilson, B.A. Harmon, M.L. McCollough, G.J. Fishman, S.N. Zhang and W.S. Paciesas, The Earth Occultation Technique with the Burst and Transient Source Experiment, in Astronomical Data Analysis Software and Systems IX, N. Manset, C. Veillet and D. Crabtree, eds., vol. 216 of Astronomical Society of the Pacific Conference Series, p. 587, Jan., 2000.

[9] J. Rodi, M.L. Cherry, G.L. Case, M.H. Finger, P. Jenke, C. Wilson et al., Earth Occultation Imaging of the Low Energy Gamma-ray Sky with GBM, in AAS/High Energy Astrophysics Division \#13, vol. 13 of AAS/High Energy Astrophysics Division, p. 117.08, Apr., 2013.

[10] A. Zoglauer, R. Andritschke and F. Schopper, MEGAlib The Medium Energy Gamma-ray Astronomy Library, 50 (2006) 629. 